\title{
La COVID-19 dans les établissements de soins de longue durée : le type de propriété joue-t-il un rôle?
}

\author{
Margaret J. McGregor MD MHSc, Charlene Harrington RN PhD
}

\author{
— Citation : CMAJ 2020 August 17;192:E961-2. doi : 10.1503/cmaj.201714-f; diffusion hâtive le 22 juillet 2020
}

Voir la version anglaise de l'article ici : www.cmaj.ca/lookup/doi/10.1503/cmaj.201714; voir la recherche connexe en anglais ici : www. cmaj.ca/lookup/doi/10.1503/cmaj.201197; voir la recherche connexe en français ici : www.cmaj.ca/lookup/doi/10.1503/cmaj.201197-f

D ans l'article associé, Stall et ses collègues se sont intéressés à la relation entre le type de propriété des établissements de soins de longue durée (SLD) et l'occurrence, l'ampleur et la mortalité associées aux éclosions de maladie à coronavirus 2019 (COVID-19) dans les 623 établissements de SLD de l'Ontario'. Dans leur analyse, ajustée à l'échelle des établissements, les auteurs n'ont trouvé aucune association entre le type de propriété et le risque d'éclosion. Ils ont toutefois constaté que les éclosions étaient plus importantes et les décès plus nombreux dans les établissements à but lucratif que dans les établissements à but non lucratif, et que la différence est encore plus marquée entre les établissements à but lucratif et les établissements dirigés entièrement par les municipalités. Malgré tout, après l'intégration du concept de chambre multiple au modèle, il n'y avait plus de différence significative, ce qui a poussé les auteurs à conclure que des rénovations aux bâtiments seraient nécessaires pour remédier aux problèmes dans les établissements de SLD en Ontario.

Dans leur modèle élargi, les auteurs ont aussi constaté que les établissements appartenant à une chaîne étaient associés à un risque significativement accru d'éclosion grave et de nombreux décès. Parmi les établissements de SLD à but lucratif de l'Ontario, $85 \%$ font partie d'une chaîne (contre $31 \%$ des établissements à but non lucratif et aucun établissement municipal), ce qui soulève la question suivante : l'appartenance des établissements de SLD à une chaîne est-elle un facteur de risque significatif et indépendant d'éclosions graves et de nombreux décès, même en tenant compte des chambres multiples? Si oui, de simples rénovations, bien qu'elles soient importantes, ne suffiront probablement pas à pallier les lacunes du système.

L'étude de Stall et ses collègues ne fournissait pas de données sur les ratios de personnel dans les différents groupes de propriétés. Dans la littérature, on mentionne toujours que la quantité d'employés en soins de longue durée est une différence importante entre les établissements d'organisations à but lucratif, ceux

\section{POINTS CLÉS}

- La recherche a montré que les foyers de soins de longue durée (SLD) à but lucratif sont associés à des éclosions et à des décès de la maladie à coronavirus 2019 (COVID-19) en Ontario; les caractéristiques des bâtiments et l'appartenance à une chaîne sont 2 facteurs de risque.

- Les organisations à but lucratif sont associées à un ratio de personnel moindre dans les recherches comparant les établissements de SLD à but lucratif et les établissements à but non lucratif.

- Des études récentes montrent un lien entre un faible ratio de personnel infirmier et l'aggravation des résultats négatifs liés à la COVID-19 dans les établissements de SLD.

- Les politiques de soins de longue durée devraient prioriser le financement et l'adoption de ratios de personnel suffisants selon les données disponibles.

d'organisations à but non lucratif et les établissements publics²,3. Une étude sur les foyers de soins de longue durée aux États-Unis publiée en 2011 a indiqué que les grandes chaînes à but lucratif offraient le moins d'heures de soins infirmiers ${ }^{4}$, et une étude canadienne antérieure a révélé que les établissements gouvernementaux (détenus par des autorités sanitaires) offraient chaque jour 61 minutes de services aux patients de plus que les établissements à but lucratif5. Un récent rapport de l'Office of the Seniors' Advocate de la Colombie-Britannique sur les contrats gouvernementaux conclus avec des établissements de SLD a montré que, de 2016 à 2018, dans le secteur à but lucratif, on a omis de fournir 207000 heures de soins pour lesquelles du financement avait été reçu, comparativement au secteur à but non lucratif, qui, lui, a fourni 80000 heures de plus que ce qui avait été financé6. En outre, la recherche commence à montrer que le ratio de personnel en SLD joue un rôle dans les éclosions de COVID-19. 
Une récente étude sur les éclosions de COVID-19 en Californie a révélé que les établissements de SLD ayant moins d'infirmières autorisées que le minimum recommandé $(0,75 \mathrm{~h}$ de soins par résident par jour) étaient 2 fois plus susceptibles de compter des résidents infectés au coronavirus du syndrome respiratoire aigu sévère 2 (SRAS-CoV-2) que les établissements qui avaient assez de personnel ${ }^{7}$. Une étude menée au Connecticut a montré que, dans les établissements de SLD comptant au moins un cas de COVID-19, chaque période supplémentaire de 20 minutes (par résident par jour) de soins par des infirmières autorisées était associée à $22 \%$ moins de cas confirmés, et, dans les établissements comptant au moins 1 décès des suites de la COVID-19, chaque période supplémentaire de 20 minutes de soins était significativement associée à $26 \%$ moins de décès ${ }^{8}$.

L'admission de l'importance des caractéristiques de l'effectif disponible a été un élément essentiel pour endiguer les éclosions de COVID-19 dans les établissements de soins de longue durée en Colombie-Britannique. On a rapidement identifié le personnel comme une source de transmission importante, ce qui a poussé le gouvernement provincial à restreindre le nombre de personnes qui travaillent dans plus d'un établissement en augmentant et en uniformisant les normes salariales et les conditions de travail pour tous les travailleurs de ce secteur ${ }^{9}$.

Ces données montrent clairement que le type de propriété influence le ratio de personnel par résident, et que ce ratio a des effets sur la gestion des éclosions de COVID-19 dans les établissements de SLD. Des données d'études des temps et mouvements et de modélisation permettent aussi de calculer facilement le nombre de personnes, par type de poste, nécessaires pour répondre aux besoins de soins des patients ${ }^{9}$. Le ratio d'un aide-soignant pour 10 résidents ou plus ne permet clairement pas de répondre aux besoins de presque tous les résidents présentement admis dans les foyers de SLD publics. Les normes minimales devraient être d'un préposé aux services de soutien à la personne (aide-soignant) pour au plus 5 à 7 résidents pour les quarts de travail de jour et de soir ${ }^{10}$. Il existe aussi des recommandations fondées sur des données probantes pour les ratios minimaux de résidents par infirmière autorisée et par infirmière auxiliaire autorisée, ajustés selon la composition des résidents ${ }^{11}$.

En préparation à la prochaine vague de COVID-19, les politiques publiques devraient viser le financement, l'adoption et l'application de ratios de personnel suffisants selon les données disponibles, non seulement pour répondre aux besoins de soins des résidents, mais aussi pour tenir compte du temps nécessaire pour les tâches liées à la sécurité, comme le lavage adéquat des mains, la mise et le retrait sécuritaires de l'équipement de protection individuelle et le respect constant des normes de lutte contre les infections. Si les exigences liées au financement d'une quantité d'employés suffisante ont des conséquences sur les résultats nets des établissements à but lucratif, il serait peut-être temps que ces soins redeviennent la responsabilité d'organisations publiques et à but non lucratif.

Comme O'Neill et ses collègues l'ont indiqué : "Si l'amélioration de la qualité augmente les coûts plus rapidement que les revenus, les profits doivent diminuer lorsque la qualité des soins augmente. Ainsi, il devrait y avoir un compromis entre qualité et profit ${ }^{12}$. » Les politiques publiques doivent accepter ce compromis et intervenir au nom des aînés les plus vulnérables.

\section{Références}

1. Stall NM, Jones A, Brown KA, et al. For-profit long-term care homes and the risk of COVID-19 outbreaks and resident deaths. CMAJ 22 juillet 2020. [Cyberpublication avant impression]. doi : 10.1503/cmaj.201714.

2. Comondore VR, Devereaux PJ, Zhou Q, et al. Quality of care in for-profit and not-for-profit nursing homes: systematic review and meta-analysis. $B M J$ 2009;339:b2732

3. Hillmer MP, Wodchis WP, Gill SS, et al. Nursing home profit status and quality of care: Is there any evidence of an association? Med Care Res Rev 2005; 62:139-66.

4. Harrington $\mathrm{C}$, Olney $\mathrm{B}$, Carrillo $\mathrm{H}$, et al. Nurse staffing and deficiencies in the largest for-profit nursing home chains and chains owned by private equity companies. Health Serv Res 2012;47:106-28.

5. McGregor MJ, Tate RB, McGrail KM, et al. Trends in long-term care facility staffing by facility ownership in British Columbia, 1996 to 2006. Health Rep 2010;21: 27-33.

6. McKenzie I. A billion reasons to care: a funding review of contracted long-term care in B.C., 2020. Victoria : Office of the Seniors Advocate; 2020. Accessible ici : https://www.seniorsadvocatebc.ca/app/uploads/sites/4/2020/02/ ABillionReasonsToCare.pdf (consulté le 15 juillet 2020).

7. Harrington C, Ross L, Chapman S, et al. Nurse staffing and coronavirus infections in California nursing homes. Policy Polit \& Nurs Pract 7 juillet 2020. [Cyberpublication avant impression]. doi : 10.1177/1527154420938707.

8. Li Y, Temkin-Greener H, Gao S, et al. COVID-19 infections and deaths among Connecticut nursing home residents: facility correlates. J Am Geriatr Soc 18 juin 2020. [Cyberpublication avant impression]. doi : 10.1111/jgs.16689.

9. COLOMBIE-BRITANNIQUE (province). Order of the Minister of Public Safety and Solicitor General. Emergency Program Act : Ministerial Order No. M105. 10 avril 2020. Accessible ici : https://www2.gov.bc.ca/assets/gov/public-safety-and-emergency -services/emergency-preparedness-response-recovery/gdx/orders-april-10/ ep_act_order_m105-2020_single_site.pdf (consulté le 12 mai 2020).

10. Schnelle JF, Schroyer LD, Saraf AA, et al. Determining nurse aide staffing requirements to provide care based on resident workload: a discrete event simulation model. J Am Med Dir Assoc 2016;17:970-77.

11. Harrington C, Dellefield ME, Halifax E, et al. Appropriate nurse staffing levels for U.S. nursing homes. Health Serv Insights 29 juin 2020. [Cyberpublication avant impression]. doi : 10.1177/1178632920934785.

12. O'Neill C, Harrington C, Kitchener M, et al. Quality of care in nursing homes: an analysis of relationships among profit, quality, and ownership. Med Care 2003;41:1318-1330.

\section{Intérêts concurrents : Aucun déclaré.}

Cet article a été commandé et a été révisé par les pairs.

Affiliations : Département de médecine familiale (McGregor), Université de la Colombie-Britannique; Centre d'épidémiologie et d'évaluation clinique (McGregor), Institut de recherche de la régie régionale de la santé Vancouver Coastal, Vancouver (ColombieBritannique); Université de la Californie à San Francisco (Harrington), San Francisco (Californie).

Collaborateurs : Les deux auteurs ont contribué à la conception du travail, ont rédigé le manuscrit et en ont révisé de façon critique le contenu intellectuel important; ils ont donné leur approbation finale pour la version destinée à être publiée et assument l'entière responsabilité de tous les aspects du travail.

Correspondance : Margaret McGregor, mrgret@mail.ubc.ca 